\title{
Motivação Para a Prática da Musculação Por Idosos do Município de Palhoça-SC
}

\author{
Gabriel de Palma Varela ${ }^{1}$, Rafaella Zulianello dos Santos ${ }^{2}$
}

\begin{abstract}
RESUMO
Introdução: Sabe-se que a expectativa de vida vem aumentando nas últimas décadas e, concomitantemente, vêm crescendo as recomendações para um estilo de vida mais saudável por parte dos idosos. Dentre estas recomendações está a prática de exercícios físicos, como a musculação, que tem chamado a atenção em razão das crescentes evidências sobre seus benefícios nesta população. Há, porém, carência de estudos na literatura que demonstrem qual a motivação dos idosos para a prática da musculação. Objetivo: Investigar qual a motivação para a prática da musculação em idosos do município de Palhoça (SC). Métodos: Pesquisa aplicada, quantitativa, do tipo descritiva, de levantamento e com corte transversal. A amostra foi do tipo não probabilística por conveniência, composta por idosos praticantes de musculação no município de Palhoça. Para análise da motivação para a prática da musculação foi aplicado o Inventário de Motivação à Prática Regular de Atividade Física e Esporte (Imprafe-54). O instrumento contém 54 perguntas divididas em seis domínios: controle de estresse, saúde, sociabilidade, competitividade, estética e prazer. Resultados: Foram avaliados 24 idosos com idade de $61,2( \pm 4,9)$ anos, praticantes de musculação a $10,3( \pm 10,7)$ anos e que frequentavam a academia em média $4,3( \pm 1,1)$ dias por semana. A dimensão que mais motiva os idosos para prática da musculação é a saúde, em detrimento da dimensão competitividade, que foi a menos citada pelos avaliados. Não houve diferença significativa entre os sexos em nenhuma dimensão. Conclusão: A principal motivação para a prática da musculação em idosos do município de Palhoça foi a dimensão saúde; já a dimensão menos motivante para esta prática é a competitividade.
\end{abstract}

Palavras-chave: Idoso. Motivação. Treinamento de resistência.

\section{MOTIVATION FOR THE RESISTANCE TRAINING PRACTICE BY ELDERLY FROM THE CITY OF PALHOÇA-SC}

\section{ABSTRACT}

Introduction: It is known that life expectancy has been increasing in the last decades and, concomitantly, the recommendations for a healthier lifestyle by the elderly. Among these recommendations is the practice of exercise, such as resistance training, which has drawn attention due to growing evidence of its benefits in this population. There is, however, a lack of studies in the literature that demonstrate the motivation of the elderly to practice of resistance training. Objective: To investigate the motivation for the practice of resistance training in the elderly in the city of Palhoça (SC). Methods: Applied, quantitative, descriptive, survey and cross-sectional research. The sample was non-probabilistic for convenience, composed of elderly people practicing resistance training in the city of Palhoça. For analysis of the motivation for the practice of resistance training was applied the Inventory of Motivation to the Regular Practice of Physical Activity and Sport (Imprafe-54). The instrument contains 54 questions divided into six domains: stress management, health, sociability, competitiveness, aesthetics and pleasure. RESULTS: Twenty-four elderly patients, mean age of $61.2( \pm 4.9)$ years, who practiced resistance training for 10.3 ( \pm 10.7 ) years and who attended the gym on average $4.3( \pm 1.1)$ days per week. The dimension that most motivates the elderly to practice resistance training is health, to the detriment of the competitiveness dimension, which was the least cited evaluated. There was no significant difference between the sexes in any dimension. Conclusion: The main motivation for the practice of resistance training in the elderly of the city of Palhoça was the health dimension; already the least motivating dimension for this practice is competitiveness.

Keywords: Elderly. Motivation. Resistance training.

Recebido em: 29/1/2019

Aceito em: $7 / 2 / 2019$

\footnotetext{
Graduado em Educação Física pela Universidade do Sul de Santa Catarina.
}

${ }^{2}$ Graduação em Educação Física pela Universidade do Estado de Santa Catarina (2004). Especialização em Exercícios Físicos para populações especiais e reabilitação cardíaca pela Universidade Gama Filho (2007). Mestrado (2009) e Doutorado (2015) em Ciências do Movimento Humano pela Universidade do Estado de Santa Catarina. Professora-adjunta do Instituto de Ensino Superior da Grande Florianópolis nos cursos de Educação Física, Nutrição e Fisioterapia, na Faculdade de Santa Catarina no curso de Estética e na Universidade do Sul de Santa Catarina no curso de Educação Física. rafaella.zulianello@gmail.com 


\section{INTRODUÇÃO}

Sabe-se que a expectativa de vida vem aumentando no Brasil em virtude do seu desenvolvimento técnico científico, em especial em áreas como a saúde. Dados do Instituto Brasileiro de Geografia e Estatística (IBGE) indicam que, em 2060, 73 milhões de brasileiros terão 60 anos ou mais (IBGE, 2018). Em razão disso, especialistas têm voltado sua atenção para o envelhecer com saúde (LI et al., 2019).

Para tanto, vêm crescendo as recomendações para um estilo de vida mais saudável por parte dos idosos (COSTA, 2017). Dentre estas recomendações está a prática de exercícios físicos que, mesmo que não consiga interromper o processo biológico de envelhecimento, tem potencial para minimizar os efeitos fisiopatológicos e a progressão de doenças crônicas relacionadas à idade (BOOTH; ROBERTS; LAYE, 2012; CAMPOS et al., 2014; FERREIRA et al., 2017).

Neste sentido, sabe-se que os níveis adequados de atividade física e aptidão física são mantidos mais facilmente quando os indivíduos se sentem motivados para a prática, percebendo efetivamente os benefícios desses comportamentos saudáveis (LOURES; KRAVCHYCHYN; TEIXEIRA, 2017). Assim, uma das conceituações clássicas define a motivação como a soma de fatores que determinam formas de comportamento voltadas a um determinado objetivo, sendo considerada o fator mais importante e determinante no que se refere ao início e continuidade de algum processo, como a prática de exercícios físicos (SAMULKI, 2009). Salienta-se, porém, que os fatores motivacionais que movem um sujeito em prol de um objetivo não são imutáveis. Ou seja, os motivos para determinada prática ou conduta podem variar ao longo do tempo de acordo com as necessidades, aspirações e obrigações do indivíduo (SAMULKI, 2009; ARO; ABGO; OMOLE, 2018).

Assim, a motivação para a prática de exercícios físicos é resultado de uma complexa interação entre diversas variáveis psicológicas, sociais, ambientais e genéticas (MARCOS-PARDO; MARTÍNEZ-RODRÍGUEZ; GIL-ARIAS, 2018). Dentre estes fatores destacam-se: o conhecimento, a atitude, o apoio social e familiar, a disponibilidade de espaço e instalações, as barreiras percebidas pela pessoa (distância, recursos, tempo) e as normas sociais (CODONHATO et al., 2017).

Dentre as modalidades de exercício físico, a musculação tem sido crescentemente procurada pelo público idoso. Assim, é instigante refletir sobre quais motivações levam este público à prática da modalidade, uma vez que as informações encontradas na lite- ratura ainda são escassas. Esses exercícios auxiliam na manutenção da boa postura, melhoram o sistema cardiorrespiratório, fortalecem a musculatura, ajudam na mobilidade, flexibilidade e autoestima, proporcionam sensação de bem-estar, alívio do estresse e controle de peso, entre tantos outros benefícios descritos (PADILLA-COLLON; SANCHEZ-COLLADO; CUEVAS, 2014; LOPEZ et al., 2018).

Uma das formas de se intervir para a continuidade de prática de exercícios físicos nas academias, portanto, é por meio da identificação dos motivos que os indivíduos atribuem a essa prática (LIZ; ANDRADE, 2016; MARCOS-PARDO; MARTÍNEZ-RODRÍGUEZ; GIL-ARIAS, 2018). Logo, os resultados do presente estudo poderão servir como um ponto de partida para a identificação das motivações dos idosos para a prática da musculação, auxiliando na elaboração de estratégias de intervenção que atendam às necessidades destes praticantes de musculação. Logo, o presente estudo objetivou investigar qual a motivação para a prática da musculação em idosos do município de Palhoça-SC.

\section{MATERIAIS E MÉTODOS}

Esta pesquisa se caracteriza como de natureza aplicada, do tipo descritiva, com abordagem quantitativa e de corte transversal. Trata-se de um estudo com amostra do tipo não probabilística por conveniência, quando o número amostral foi estabelecido com base em uma consulta prévia às cinco academias do município de Palhoça, onde as coletas foram realizadas.

Como critérios de inclusão foram estabelecidos: ter 60 anos ou mais, ser praticante de musculação no município de Palhoça por pelo menos três meses e assinar do Termo de Consentimento Livre e Esclarecido. Como critério de exclusão foi estabelecido não preencher pelo menos $80 \%$ do instrumento.

O projeto foi aprovado pelo Comitê de Ética em Pesquisa em Seres Humanos da Universidade do Sul de Santa Catarina, sob o número 2.296.791, de acordo com a resolução 466/12 do Conselho Nacional de Saúde. Para caracterização da amostra foi utilizada uma anamnese com informações sobre doenças prévias, nível socioeconômico e informações sobre a atividade física rotineira. Para avaliação da motivação para a prática da musculação utilizou-se o "Inventário de Motivação à Prática Regular de Atividade Física e Esporte" (Imprafe-54), criado por Balbinotti e Barbosa (2008). O Imprafe-54 tem o objetivo de conhecer as motivações que levam à prática de atividades físicas, construído para atender pessoas entre 13 e 83 anos. São 54 perguntas, divididas nas dimensões controle 
de estresse, saúde, sociabilidade, competitividade, estética e prazer. Cada questão é pontuada por uma escala bidirecional, de tipo Likert, dividida em cinco pontos, posto que o número um é o que motiva pouquíssimo e o cinco que motiva muitíssimo (BALBINOTTI; BARBOSA, 2008).

Primeiramente foram contatadas as academias de musculação no município de Palhoça para a explanação da pesquisa e solicitação de autorização para realização da mesma nas suas dependências. Logo, o pesquisador combinou e marcou dias e horários com os respectivos responsáveis para a realização da pesquisa. As coletas de dados foram feitas entre os meses de março e abril de 2018.

Para análise dos dados foi utilizada estatística descritiva com média e desvio padrão para as variáveis contínuas e frequência simples (f) e relativa (\%) para as variáveis categóricas. Para a normalidade dos dados utilizou-se o teste de Kolmogorov-Smirnov. Para comparar os motivos para a prática da musculação entre homens e mulheres idosos, utilizou-se o teste $U$ de Mann Whitney, em razão da não normalidade dos dados. $O$ nível de significância adotado foi de 5\%. Para todas as análises foi utilizado o software Statistical Package for the Social Sciences (SPSS) versão 20.0.

\section{RESULTADOS}

Foram identificados 32 idosos com potencial para participar do estudo e, destes, 24 aceitaram responder ao instrumento. Dois não aceitaram participar do estudo por motivos pessoais e seis não foram encontrados nas academias durante a realização do estudo. Sendo assim, a amostra final da presente pesquisa foi de 24 idosos com média de idade de $61,2( \pm 4,9)$ anos. Os participantes da pesquisa relataram praticar musculação a 10,3 $( \pm 10,7)$ anos e frequentar a academia para tal prática em média $4,3( \pm 1,1)$ dias por semana. As características destes participantes são apresentadas na Tabela 1.

Tabela 1 - Características socioeconômicas e clínicas dos idosos da pesquisa $(n=24)$

\begin{tabular}{llcc}
\hline Variável & Classe & f & \% \\
\hline \multirow{3}{*}{ Sexo } & Masculino & 13 & 54,2 \\
& Feminino & 11 & 45,8 \\
\cline { 2 - 4 } & Solteiro & 1 & 4,2 \\
& Casado & 14 & 58,3 \\
& Divorciado & 5 & 20,3 \\
& Viúvo & 3 & 12,5 \\
& União estável & 1 & 4,2 \\
\cline { 2 - 4 } & & &
\end{tabular}

\begin{tabular}{llcc}
\multirow{4}{*}{ Escolaridade } & Fundamental incompleto & 4 & 16,7 \\
& Fundamental Completo & - & - \\
& Médio Incompleto & 1 & 4,2 \\
& Médio Completo & 9 & 37,5 \\
& Superior Incompleto & 3 & 12,5 \\
& Superior Completo & 5 & 20,8 \\
Renda & Pós-Graduação & 2 & 8,3 \\
\cline { 2 - 4 } familiar & Até 1 Salário & 2 & 8,3 \\
& Entre 1 e 5 Salários & 9 & 37,5 \\
& Entre 5 e 10 Salários & 11 & 45,8 \\
Doenças & Mais que 10 salários & 2 & 8,3 \\
\cline { 2 - 4 } associadas & DAC & 3 & 12,3 \\
& Dislipidemias & 3 & 12,5 \\
& DM & 3 & 12,5 \\
& DAOP & 1 & 4,2 \\
& AVE & 1 & 4,2 \\
& Tabagismo & 6 & 25 \\
& IM & 2 & 8,3 \\
& HAS & 3 & 12,5 \\
& Problemas osteomioarticulares & 3 & 12,5 \\
\hline
\end{tabular}

$\mathrm{f}$ - frequência simples; \% - frequência relativa; DAC - Doença arterial Coronariana; DM - Diabetes mellitus; DAOP - Doença arterial obstrutiva Periférica; AVE - Acidente Vascular encefálico; IM - Infarto do miocárdio; HAS - Hipertensão arterial sistêmica.

Fonte: Elaborada pelos autores.

A Tabela 2 mostra as seis dimensões relacionadas com a motivação que levam os idosos a realizar a musculação.

Tabela 2 - Dimensões motivacionais para a prática de musculação pelos idosos do estudo $(n=24)$

\begin{tabular}{lcc}
\hline Dimensão & Média & DP \\
\hline Saúde & 35,5 & 2,6 \\
Competitividade & 8,9 & 3,5 \\
Estética & 33,7 & 5,5 \\
Sociabilidade & 31,8 & 9,8 \\
Controle do estresse & 33,5 & 5,9 \\
Prazer & 31,3 & 3,9 \\
\hline
\end{tabular}

DP - Desvio padrão.

Fonte: Elaborada pelos autores.

A Tabela 3 mostra que, quando comparadas as dimensões motivacionais entre homens e mulheres idosas, não houve diferença significativa entre nenhuma dimensão. A ordem das dimensões motivacionais também foi a mesma para homens e mulheres: saúde seguido pelo controle de estresse, estética, sociabilidade, prazer e competitividade.

Tabela 3 - Comparação das motivações para a prática da musculação entre homens e mulheres idosas ( $n=24)$

\begin{tabular}{lccc}
\hline Dimensão & Homens & Mulheres & $\boldsymbol{P}$ \\
\cline { 2 - 3 } & Média (DP) & Média (DP) & \\
\hline Saúde & $38,1( \pm 2,9)$ & $39( \pm 2,1)$ & 0,665
\end{tabular}




\begin{tabular}{lccc} 
Competitividade & $9,4( \pm 4,7)$ & $8,4( \pm 1,2)$ & 0,6550 \\
Estética & $32,9( \pm 6,2)$ & $34,5( \pm 4,6)$ & 0,380 \\
Sociabilidade & $31,4( \pm 10,3)$ & $32,6( \pm 9,8)$ & 0,839 \\
Controle do & $35,5( \pm 6,8)$ & $37,6( \pm 4,6)$ & 0,540 \\
estresse & & & \\
Prazer & $30,2( \pm 4,8)$ & $32,5( \pm 2,1)$ & 0,649 \\
\hline
\end{tabular}

DP - Desvio padrão; $p$ - significância estatística para o teste $U$ de Mann Whitney entre os sexos $<0,05$.

Fonte: Elaborada pelos autores.

\section{DISCUSSÃO}

O presente estudo objetivou mostrar qual a motivação para a prática da musculação em idosos do município de Palhoça-SC. A análise de variáveis motivacionais para a prática da musculação em idosos tem despertado interesse, visando a identificar os principais motivadores deste grupo especial a fim de melhorar a adesão ao programa de treinamento de força (MARCOS-PARDO; MARTÍNEZ-RODRÍGUEZ; GIL-ARIAS, 2018). Neste sentido, dentre os principais achados deste trabalho destaca-se que a dimensão estudada que mais motiva os idosos para a prática da musculação foi a saúde, em detrimento da dimensão competitividade que foi a menos citada pelos avaliados. Concordando com estes achados, um estudo, realizado na cidade do Recife com 120 idosos de programas de saúde ligados à rede pública, avaliados por um questionário adaptado sobre motivação para a prática esportiva, observou que fatores relacionados à saúde e ao desempenho físico também foram motivos importantes entre os idosos, tanto para começar ou para permanência nas atividades físicas de forma geral (FREITAS et al., 2007).

Ainda corroborando com estes resultados, outro trabalho comparou os fatores motivacionais para prática de atividades físicas gerais entre idosos brasileiros e portugueses, com amostra predominantemente feminina nos dois países $\mathrm{A}$ saúde foi percebida como uma preocupação comum para ambas as realidades culturais (CAVALLI et al., 2014). De forma semelhante, os resultados encontrados no estudo de Stiggelbout, Hopman-Rock e Mechelen (2008) indicam a melhoria da saúde como o principal fator motivacional para idosos participantes de programas de atividades físicas.

Outro estudo que aplicou o mesmo inventário em idosos apresentou que a dimensão saúde foi a mais motivadora para a prática de atividades físicas, seguida de prazer e sociabilidade (MEURER; BENEDETTI; MAZO, 2012). No mesmo sentido, o estudo de Meurer (2010), avaliando 140 idosos que partici- pavam de um programa de atividades físicas em Florianópolis, mostrou que a saúde, o prazer e a sociabilidade são, respectivamente, os aspectos que mais motivam os idosos a frequentar algum tipo de atividade física, enquanto a competitividade e a estética são os que menos levam esses idosos a realizarem atividades físicas. Estes achados diferem em partes dos do presente trabalho, uma vez que, ainda que a saúde também tenha sido o fator mais motivante para a prática da musculação, esta foi seguida de perto pelo controle do estresse e estética, respectivamente.

Quando se fala na motivação de idosos especificamente para a musculação, foram encontrados poucos estudos (MARCOS-PARDO; MARTÍNEZ-RODRÍGUEZ; GIL-ARIAS, 2018). O trabalho de Cardoso (2014) utilizando o Impraf-54, porém, mostrou que a ordem dos fatores motivacionais foi muito parecida com o presente trabalho, uma vez que a saúde se destacou como o principal fator motivacional, e o menos importante foi a competitividade.

Ao serem comparadas as dimensões motivacionais entre homens e mulheres idosas, não houve diferença significativa entre nenhuma dimensão. A ordem das dimensões motivacionais foi a mesma para homens e mulheres: saúde, seguida pelo controle de estresse, estética, sociabilidade, prazer e competitividade. Quando comparado este estudo a outro com objetivos parecidos, pode-se notar que o principal motivo, tanto para homens quanto para mulheres que buscam a prática em academias, foi a saúde, seguido por prazer, estética, controle de estresse, sociabilidade e competitividade (BALBINOTTI; CAPOZZOLLI, 2008). Zawadski e Vagetti (2007) analisaram 24 mulheres com mais de 60 anos de idade e constataram que os motivos que as levavam para a prática da musculação foram a "Prevenção de doenças e manutenção à saúde", com $42 \%$ dos resultados, seguido da "Indicação Médica e fazer amigos", com $25 \%$ dos resultados cada. Ou seja, assim como no presente estudo, as senhoras preocupam-se com a saúde, mas também levam em consideração a socialização. A razão, todavia, que menos levou os idosos às academias foi a dimensão "competitividade", tanto para homens quanto para mulheres.

Corroborando estes achados está o estudo de Ferreira, Souza e Araújo (2012), que foi feito com 10 homens e 10 mulheres entre 60 e 77 anos participantes atividades físicas em Brasília. $O$ estudo observou que a dimensão competitividade também foi a menos citada como motivadora da prática em ambos os sexos. 
Neste quesito competitividade estão inseridos fatores como disputa, resultado, avaliação, seleção, ímpeto pela vitória, desprezo pela derrota, pressão, alegria e frustração (DE ROSE JUNIOR; DE CAMPOS; TRIBST, 2001). Possivelmente estes não são anseios primordiais dos idosos, uma vez que boa parte destes possivelmente se encontram aposentados e com objetivos de vida diferentes dos jovens, nos quais a competitividade impera na vida tanto profissional quanto social.

É importante notar, portanto, que, para que a população idosa venha a integrar exercícios físicos regulares em suas rotinas, em especial a musculação, ela deve não apenas conhecer os benefícios dessa prática, mas também as sessões devem ser elaboradas com base em suas necessidades específicas, bem como os exercícios ajustados ao seu nível de competência. Além disso, os exercícios devem ser realizados de forma que enalteça as expectativas destes grupos para que o componente motivacional possa atuar como fator determinante no desempenho e continuidade da prática da musculação (MARCOS-PARDO; MARTÍNEZ-RODRÍGUEZ; GIL-ARIAS, 2018).

É necessário, porém, cautela na interpretação destes resultados. Em primeiro lugar é preciso destacar que a motivação dos sujeitos é muito subjetiva e pode se apresentar distinta em diferentes cortes no tempo para a mesma amostra estudada. Em segundo lugar o número amostral é limitado, e isso se deve às limitações de tempo para a realização da pesquisa e de disponibilidade de participação dos convidados, o que reduz o alcance dos dados. Em terceiro lugar, em razão de o estudo ser de caráter transversal, sendo as análises realizadas num ponto específico do tempo, o alcance dos resultados também é limitado.

\section{CONCLUSÃO}

A principal motivação para a prática da musculação em idosos do município de Palhoça foi a dimensão saúde; já a dimensão menos motivante para esta prática foi a competitividade. Não houve diferença significativa entre as motivações de homens e mulheres idosas, sendo também a dimensão saúde a mais motivante e a dimensão estética a menos motivante. São poucos os estudos, entretanto, que avaliam a motivação para a prática da musculação especificamente para os idosos. A maioria fala de exercício físico genericamente; logo, sugere-se que sejam realizados outros estudos com idosos de diferentes regiões e, especificamente, observando a motivação para a prática da musculação.

\section{REFERÊNCIAS}

ARO, A. A.; AGBO, S.; OMOLE, O. B. Factors influencing regular physical exercise among the elderly in residential care facilities in a South African health district. Afr J Prim Health Care Fam. Med., v. 10, n. 1, e1-e6, 2018.

BALBINOTTI, M. A. A.; BARBOSA, M. L. L. Manual de aplicação do inventário motivação à prática regular de atividades físicas (IMPRAF - 126). Universidade Federal do Rio Grande do Sul: Porto Alegre, 2006.

BALBINOTTI, M. A. A.; CAPOZZOLI, C. J. Motivação à prática regular de atividade física: um estudo exploratório com praticantes em academias de ginástica. Revista Brasileira de Educação Física Especial, São Paulo, v. 22, n. 1, p. 63-80, jan./mar. 2008. Disponível em: https://www.revistas.usp. br/rbefe/article/download/16683/18396/. Acesso em: 28 abr. 2018.

BOOTH, F. W.; ROBERTS, C. K. LAYE, M. J. Lack of exercise is a major cause of chronic diseases. Compr Physiol. Apr., v. 2, n. 2, p. 1.143-1.211, 2012.

CAMPOS, A. C. V. et al. Qualidade de vida de idosos praticantes de atividade física no contexto da estratégia saúde da família. Texto Contexto Enferm., v. 23, n. 4, p. 889-897, 2014.

CARDOSO, A. M. N. Fatores motivacionais de idosos associados à prática regular de exercícios físicos. 2014, 122f. Dissertação (Mestrado) - Universidade Estadual Paulista, Instituto de Biociências, Rio Claro, SP, 2014.

CAVALLI, A. S. et al. Motivation of elderly people to engage in physical exercising: a comparative study between two university-based programs Brazil and Portugal. Rev. Bras. Geriatr. Gerontol., v. 17, n. 2, p. 255-264, 2014.

CODONHATO, R. et al. Qualidade de vida e motivação de praticantes de exercício físico. Corpoconsciência, v. 21, n. 1, p. 92-99, 2017.

COSTA, A. S. Estratégia nacional para o envelhecimento ativo e saudável 2017-2025, 2017. Disponível em: https:// www.sns.gov.pt/wp-content/uploads/2017/07/ENEAS.pdf. Acesso em: 7 fev. 2018.

DE ROSE JUNIOR, D.; DE CAMPOS, R. R.; TRIBST, M. Motivos que llevan a la práctica del baloncesto: un estudio con jóvenes atletas brasileños. Revista de Psicologia del Deporte, Barcelona, v. 10, n. 2, p. 293-304, 2001.

FERREIRA, D. A.; SOUZA, J. R.; ARAÚJO, M. J. S. Motivações e perfil socioeconômico e cultural dos frequentadores de academias em Picos. In: FÓRUM INTERNACIONAL DE PEDAGOGIA, 4., Parnaíba, Campina Grande: Realize, 2012.

FERREIRA, M. C. G. et al. Representações sociais de idosos sobre qualidade de vida. Rev. Bras. Enferm., v. 70, n. 4, p. 840-847, 2017.

FREITAS C. M. S. M. et al. Aspectos motivacionais que influenciam a adesão e manutenção de idosos a programas de exercícios físicos. Rev. Bras. Cineantropom Desempenho Humano, v. 9, n. 1, p. 92-100, 2007. 
IBGE. Instituto Brasileiro de Geografia e Estatística. Projeções da população. 2018. Disponível em: https://www. ibge.gov.br/estatisticas/sociais/populacao/9109-projecao-da-populacao.html?=\&t=downloads. Acesso em: 30 maio 2019.

LI, J. et al. Spatiotemporal evolution of global population ageing from 1960 to 2017. BMC Public Health, v. 19, n. 1, p. 127, 2019.

LIZ, C. M.; ANDRADE, A. Análise qualitativa dos motivos de adesão e desistência da musculação em academias. Rev. Bras. Ciênc. Esporte, v. 38, n. 3, p. 267-274, 2016.

LOPEZ, P. et al. Benefits of resistance training in physically frail elderly: a systematic review. Aging Clinical and Experimental Research, v. 30, n. 8, p. 889-899, 2018.

LOURES, R. R. R.; KRAVCHYCHYN, C.; TEIXEIRA, R. T. S. Motivação para o exercício físico em idosos de diferentes classes sociais. Coleção Pesquisa em Educação Física, v. 16, n. 2, p. 87-94, 2017.

MARCOS-PARDO, P. J.; MARTÍNEZ-RODRÍGUEZ, A.; GILARIAS, A. Impact of a motivational resistance-training programme on adherence and body composition in the elderly. Sci Rep., v. 8, p. 1.370, 2018.
MEURER, S. T.; BENEDETTI, T. R. B.; MAZO, G. Z. Fatores motivacionais de idosos praticantes de exercícios físicos: um estudo baseado na teoria da autodeterminação. Estudos de Psicologia, v. 17, n. 2, p. 299-304, 2012.

MEURER, S. T. Motivação para a prática de exercícios físicos, autoestima e aptidão funcional de idosos: interpretações baseadas na teoria da autodeterminação. 2010. 140 f. Dissertação (Mestrado) - Universidade Federal de Santa Catarina, Curso de Educação Física, Florianópolis, 2010.

PADILLA-COLLON, C. J.; SANCHEZ-COLLADO, P.; CUEVAS, M. $J$. Benefits of strength training for the prevention and treatment of sarcopenia. Nutrición Hospitalaria, v. 29, n. 5, p. 979-988, 2014.

SAMULSKI, D. M. Motivação. In: SAMULSKI, D. M. Psicologia do esporte: conceitos e novas perspectivas. 2. ed. Barueri: Manole, 2009. p. 167-192. Cap. 2.

STIGGELBOUT, M.; HOPMAN-ROCK, M.; MECHELEN, W. Entry correlates and motivations of older adults participating in organized exercise programs. Journal of Aging and Physical Activity, v. 16, n. 3, p. 342-354, 2008.

ZAWADSKI, A. B. R.; VAGETTI, G. C. Motivos que levam idosas a frequentarem musculação. Revista Movimento \& Percepção, Espírito Santo do Pinhal, n. 10, p. 45-60, 2007. 